\title{
FINITE ELEMENT APPROXIMATIONS OF A GLACIOLOGY PROBLEM
}

\author{
Sum S. Chow ${ }^{1}$, Graham F. Carey ${ }^{2}$ and Michael L. Anderson ${ }^{2}$
}

\begin{abstract}
In this paper we study a model problem describing the movement of a glacier under Glen's flow law and investigated by Colinge and Rappaz [Colinge and Rappaz, ESAIM: M2AN 33 (1999) 395406]. We establish error estimates for finite element approximation using the results of Chow [Chow, SIAM J. Numer. Analysis 29 (1992) 769-780] and Liu and Barrett [Liu and Barrett, SIAM J. Numer. Analysis 33 (1996) 98-106] and give an analysis of the convergence of the successive approximations used in [Colinge and Rappaz, ESAIM: M2AN 33 (1999) 395-406]. Supporting numerical convergence studies are carried out and we also demonstrate the numerical performance of an a posteriori error estimator in adaptive mesh refinement computation of the problem.
\end{abstract}

Mathematics Subject Classification. 26B25, 35J20, 35J60, 49J45, 65N30, 86A40.

Received: April 22, 2003. Revised: June 18, 2004.

\section{INTRODUCTION}

There has been a longstanding interest in modeling the flow of glaciers in the environmental science community. Most modern models are based on the underlying conservation laws of mechanics together with constitutive relationships describing the behavior of ice. There are numerous complications both at the level of the constitutive relation and also in the treatment of the sliding base and the growth and melting of the ice layer. For a general introduction to the problem of modeling the flow of glaciers and the associated constitutive relations, see, for example, Paterson [13]. Of particular interest in the present work are models based on the well-established Glen's flow law for polychrystaline ice [8] in which the shear strain rate is proportional to the shear stress raised to a power $n$, and where the proportionality factor depends on ice temperature, crystal size, impurity content and other factors.

In [7], Colinge and Rappaz considered a model 2D problem for the movement of a glacier under Glen's flow law and established the existence and uniqueness of the weak solution to the corresponding boundary value problem. They further proved the convergence of the finite element approximations and numerically studied the convergence of the successive approximation method. However, no error estimates for the finite element approximations and theoretical convergence analysis of the method of successive approximation were given.

In this paper we show that by considering the modeling equation in a slightly different but equivalent form, it is possible to establish error estimates for the finite element approximations using the results of Chow [4] and Liu and Barrett [12]. As a consequence of the analysis, a uniform boundedness result for the gradient of the finite element approximations may be established. This in turn allows us to provide an analysis of the

Keywords and phrases. Glen's flow law, non-Newtonian fluids, finite element error estimates, successive approximations.
1 Department of Mathematics, Brigham Young University, Provo, UT 84602 , USA. e-mail: schow@math.byu.edu
2 ICES, Univ. of Texas at Austin, Austin, TX 78712, USA. e-mail: carey@cfdlab.ae.utexas.edu; michaela@rsp.com.au

(c) EDP Sciences, SMAI 2004 
convergence of the successive approximation used in [7]. In the last section, a finite element implementation of the model and supporting numerical studies are included to evaluate rate of convergence for a well-behaved solution and in the presence of of a partial-slip base. Adaptive mesh refinement studies are also carried out in the latter case to treat the stick-slip singularity and to improve accuracy and efficiency.

Numerically determined convergence rates for a two problems are compared with the theoretical convergence rates for uniform refinement. Also convergence rates for adaptive refinement schemes are considered.

\section{Glacier MOtion}

In the study of the movement of a glacier it is useful to treat the ice as an incompressible viscous nonNewtonian fluid and model the motion in terms of mass conservation, linear momentum balance and nonlinear stress-strain relation. In glaciology, the nonlinear stress-strain relation that characterizes the non-Newtonian nature of the fluid flow is commonly expressed by Glen's flow law [8]

$$
F(T)=A\left(T_{0}^{2}+T^{2}\right)^{\frac{n-1}{2}},
$$

where $A$ is a temperature dependent parameter and is a constant under assumption of isothermal condition, $T_{0}$ is a positive constant, $n>1$ is a positive integer and $T$ is the second invariant of the deviatoric stress tensor $\sigma_{i j}$, which is related to the symmetric Cauchy stress tensor $\tau_{i j}$ via $\sigma_{i j}=\tau_{i j}-\frac{1}{3} \tau_{k k} \delta_{i j}$. Note that other forms of flow laws are also possible. For example, a flow law that is valid over a wider stress range was listed in [13], p. 27 ) and is related to the Eyring model of non-Newtonian fluid. For other studies on the Eyring model, see [5].

As the modeling equations are fairly complex, various simplifying assumptions are usually made to arrive at a more basic model for analysis $[1,13]$. For example, in the model considered here, a glacier sliding down a plane with a small constant angle of inclination $\alpha$, the ice slab is assumed to have parallel flat upper and lower surfaces and thus a constant thickness $\mathcal{H}$. As the aspect ratio $\epsilon=\mathcal{H} / \mathcal{L}$ of the thickness $\mathcal{H}$ of an ice sheet to its extent $\mathcal{L}$ is small, it may be used as a scaling parameter.

Let the Cartesian coordinate system $(x, y, z)$ be chosen so that the $z$-axis is opposite to gravity. Let $\mathcal{U}, \mathcal{V}, \mathcal{W}$ be the corresponding velocity components. By non-dimensionalizing the governing equations in $\epsilon$, and denoting the scaled variables by the original symbols for notational convenience, after dropping all terms of order $\mathcal{O}\left(\epsilon^{2}\right)$, a set of eight equations [1] for the variables $\sigma_{x x}, \sigma_{y y}, \tau_{x y}, \tau_{y z}, \tau_{z x}, \mathcal{U}, \mathcal{V}, \mathcal{W}$ is obtained from mass conservation,

$$
\epsilon \frac{\partial W}{\partial z}=-F(T)\left(\sigma_{x x}+\sigma_{y y}\right),
$$

momentum balance,

$$
\begin{aligned}
& \epsilon\left(2 \frac{\partial \sigma_{x x}}{\partial x}+\frac{\partial \sigma_{y y}}{\partial x}+\frac{\partial \tau_{x y}}{\partial y}\right)+\frac{\partial \tau_{x z}}{\partial z}=\frac{\partial S_{T}}{\partial x}, \\
& \epsilon\left(2 \frac{\partial \sigma_{y y}}{\partial y}+\frac{\partial \sigma_{x x}}{\partial y}+\frac{\partial \tau_{x y}}{\partial x}\right)+\frac{\partial \tau_{y z}}{\partial z}=\frac{\partial S_{T}}{\partial y}
\end{aligned}
$$

where $S_{T}$ is a function describing the top surface of the ice slab, and constitutive equations

$$
\begin{gathered}
\epsilon \frac{\partial \mathcal{U}}{\partial x}=F(T) \sigma_{x x}, \quad \epsilon \frac{\partial \mathcal{V}}{\partial y}=F(T) \sigma_{y y}, \\
\frac{\partial \mathcal{U}}{\partial z}+\epsilon^{2} \frac{\partial \mathcal{W}}{\partial x}=2 F(T) \sigma_{x z}, \\
\frac{\partial \mathcal{V}}{\partial z}+\epsilon^{2} \frac{\partial \mathcal{W}}{\partial y}=2 F(T) \sigma_{y z} \\
\epsilon\left(\frac{\partial \mathcal{U}}{\partial y}+\frac{\partial \mathcal{V}}{\partial x}\right)=2 F(T) \sigma_{x y} .
\end{gathered}
$$


We assume that the ice slab has constant thickness and that the ice flow is parallel to the $x z$-plane. We then have $\mathcal{V}=\tau_{y z}=\tau_{z x}=0$. Further simplification is achieved by applying a plane-strain approximation, which states that all quantities have no dependency in the $y$ variable. The plane strain approximation reduces the effective dimension of the problem to two. This corresponds to a slice through a slab that is infinite in the $y$-direction.

We now reorient the $x$-axis so that it lies parallel to the slab direction and the $z$-axis is perpendicular to the slab. The region of interest is described by the bounded rectangular domain $(0, L) \times(0,1)$ in the $x z$-plane, where $L$ denotes a scaled horizontal length. Let $\tau$ and $\sigma$ denote the shear stress and the $x$-component of the deviatoric stress tensor respectively. By normalizing the thickness and further scaling of the independent and dependent variables, the momentum balance equations and stress-strain relation (2)-(8) are simplified to the following nonlinear modeling equation:

$$
-\frac{\partial}{\partial x}\left(\frac{1}{F(\sigma, \tau)} \frac{\partial v}{\partial x}\right)-\frac{\partial}{\partial z}\left(\frac{1}{F(\sigma, \tau)} \frac{\partial v}{\partial z}\right)=\frac{1}{2}
$$

over the domain $\Omega=(0, L) \times(0,2)$, where $v(x, z)=\mathcal{U}(x, z / 2)$.

Note that the dependence on the angle of inclination is accommodated through the scaling and hence does not appear explicitly in the governing equations. For further details, we refer the reader to $[1,6]$. The stress-strain relation now takes the form

$$
\begin{gathered}
\sigma=\frac{1}{F(\sigma, \tau)} \frac{\partial v}{\partial x} \\
\tau=\frac{1}{F(\sigma, \tau)} \frac{\partial v}{\partial z}
\end{gathered}
$$

and the Glen's flow law (1) becomes

$$
F(\sigma, \tau)=\left(T_{0}^{2}+\tau^{2}+\sigma^{2}\right)^{\frac{n-1}{2}} .
$$

Once $v$, and thus $\mathcal{U}$, are determined, the $z$-component of the velocity $\mathcal{W}$ may be obtained from the incompressibility condition (2). Consequently, we shall focus on the solution for the function $v$.

For simplicity, we assume a zero stress boundary condition on the upper surface and prescribed velocities on other surfaces:

$$
\begin{aligned}
\frac{\partial v}{\partial \nu} & =0 & & \text { on } \partial \Omega \backslash \Gamma \\
v(x, z) & =g(x, z / 2) & & \text { on } \Gamma
\end{aligned}
$$

where $\Gamma$ is a portion of $\partial \Omega$ with nonzero measure and $\nu$ denotes the outward unit normal.

Colinge and Rappaz represented $F(\sigma, \tau)$ in $[7]$ as $f\left(|\nabla v|^{2}\right)$ and showed that the modeling problem has an associate minimization problem with the corresponding functional being strictly convex and weak lower semicontinuous and hence admits a unique solution.

By representing $F(\sigma, \tau)$ in a slightly different form, namely $1 / k(|\nabla v|)$, we show that one can employ results of Chow [4] to recover the existence and uniqueness and convergence results in [7] and also additionally obtain error estimates for the finite element approximations as follows.

Rewriting (11) and (10) in terms of a vector $\Phi=(\sigma, \tau)^{T}$, Glen's law (12) becomes $F(s)=\left(|\Phi|^{2}+T_{0}^{2}\right)^{\frac{n-1}{2}}$, and we have

$$
F(|\Phi|) \Phi=\nabla v .
$$

Taking the modulus on both sides and writing $t=|\nabla v|$ and $s=|\Phi|$, we have $F(s) s=t$. 
As $F(s) s$ is strictly increasing for $s \geq 0$, it has an inverse, which we shall denote by $k(\cdot) \cdot$, i.e. $k(t) t=$ $s$ whenever $F(s) s=t$. We note that $k(t) \cdot F(s)=1$ and

$$
\frac{\mathrm{d} F(s)}{\mathrm{d} s}=(n-1)\left(s^{2}+T_{0}^{2}\right)^{\frac{n-3}{2}} s \geq 0, \quad \text { for } s \geq 0
$$

so $F$ is an increasing function of $s$ and hence $k$ is a decreasing function of $t$.

The modeling problem (11)-(13) is then equivalent to:

$$
\begin{array}{ll}
-\nabla \cdot(k(|\nabla v|) \nabla v)=\frac{1}{2} & \text { in } \Omega \\
v(x, z)=g(x, z / 2) & \text { on } \Gamma \\
\frac{\partial v}{\partial \nu}=0 & \text { on } \partial \Omega \backslash \Gamma .
\end{array}
$$

Let $G(x, z)$ be a sufficiently smooth function defined in $\Omega$ such that its trace is equal to $g(x, z / 2)$ on $\Gamma$. Setting $v=u+G$, the boundary value problem (15)-(17) may then be written as: find $u(x, z)$ such that

$$
\begin{aligned}
-\nabla \cdot(k(|\nabla(u+G)|) \nabla(u+G)) & =f & & \text { in } \Omega \\
u(x, z) & =0 & & \text { on } \Gamma \\
\frac{\partial(u+G)}{\partial \nu} & =0 & & \text { on } \partial \Omega \backslash \Gamma
\end{aligned}
$$

where $f=\frac{1}{2}$.

Let $p=1+\frac{1}{n}$, where $n$ is the Glen's flow law index. As $n \geq 1$, then $1<p \leq 2$. Define

$$
V=\left\{w \in W^{1, p}(\Omega) ; w=0 \text { on } \Gamma\right\}
$$

equipped with norm $\|\cdot\|=\|\cdot\|_{W_{0}^{1, p}(\Omega)}$ and let $V^{*}$ denote its dual with norm $\|\cdot\|^{*}$.

Assuming $g(x, z / 2)$ lies in the trace space $W^{1-1 / p, p}(\Gamma)$, we have an extension $G(x, z) \in W^{1, p}(\Omega)$. The weak formulation of (18)-(20) may then be posed as:

Find $u \in V$ such that

$$
(\mathcal{A} u, w)=(f, w) \text { for all } w \in V
$$

where $\mathcal{A}: V \rightarrow V^{*}$ is the operator defined by

$$
(\mathcal{A} u, w)=\int_{\Omega} k(|\nabla(u+G)|) \nabla(u+G) \cdot \nabla w \mathrm{~d} x \mathrm{~d} z
$$

and $(f, w)$ denotes the usual $L^{2}$-inner product of $f$ and $w$ over $\Omega$.

The corresponding minimization problem is given by:

Find $u \in V$ such that

$$
J(u) \leq J(w) \text { for all } w \in V
$$

where $J: V \rightarrow \mathbb{R}$ denotes the functional

$$
J(w)=\frac{1}{2}\left(\int_{\Omega} \int_{0}^{|\nabla(w+G)|} k(t) t \mathrm{~d} t-f w\right) \mathrm{d} x \mathrm{~d} z .
$$

Let $V_{h} \subset V$ be a conforming finite element subspace of piecewise continuous polynomials of degree $m$ over a triangulation $\mathcal{T}_{h}$ of $\Omega$ parameterized by $h>0$. The finite element approximation $u_{h} \in V_{h}$ to the weak solution 
$u \in V$ of $(21)$ is obtained by solving

$$
\left(\mathcal{A} u_{h}, w_{h}\right)=\left(f, w_{h}\right) \text { for all } w_{h} \in V_{h} .
$$

Equivalently, the finite element solution is the minimizer of the functional $J$ over $V_{h}$ :

$$
J\left(u_{h}\right)=\min _{v_{h} \in V_{h}} J\left(v_{h}\right) .
$$

\section{FunCtion PROPERTIES}

In $[3,4]$ a problem with similar structure to (18)-(20) was considered. For the results in the cited papers to be applicable, we need to show that for some constant $p$ in $(1,2]$,

(i) $k(t) \geq 0$ for $t \geq 0$ with upper bound $\hat{k}>0$;

(ii) $k(t) t$ is strictly increasing and vanishes at $t=0$;

(iii) $k(t) t$ is Hölder continuous with exponent $p-1$;

(iv) $k(t) t$ is continuously differentiable for all $t>0$, and satisfies the monotonicity inequality

$$
\left(K_{1}+K_{2} t^{2-p}\right) \frac{\mathrm{d}}{\mathrm{d} t}(k(t) t) \geq C_{1}>0 \text { for all } t>0
$$

where $K_{1}, K_{2}$ and $C_{1}$ are positive constants.

In our context, we set $p=1+1 / n$ and proceed to verify the above properties by examining corresponding properties of $F(s)$, as we do not have an explicit form for $k(t)$. More precisely, we need to establish that (see $[4])$ :

(a) There exist constants $\alpha_{1}>0, \alpha_{2} \geq 0$ such that $F(s)$ is bounded below and

$$
F(s) s \geq\left(\left(s+\alpha_{2}\right) / \alpha_{1}\right)^{n-1} \quad \text { for all } s \geq 0 .
$$

(b) $F(s) s$, the inverse of $k(t) t$, is a strictly increasing function of $s \geq 0$.

(c) There exists a constant $\gamma>0$ such that

$$
\frac{\mathrm{d}}{\mathrm{d} s}(F(s) s)^{1 / n} \geq \frac{1}{\gamma} \text { for all } s>0 .
$$

(d) The function $F(s) s$ is continuously differentiable for each $s \geq 0$ and there exist constants $C_{1}>0, C_{2}>0$ such that for all $s>0$,

$$
\frac{\mathrm{d}}{\mathrm{d} s}(F(s) s) \leq C_{1}+C_{2}(F(s) s)^{2-p} .
$$

For Glen's flow law (12), $F(s)=A\left(s^{2}+T_{0}^{2}\right)^{\frac{n-1}{2}}$. For simplicity we take the constant $A=1$ in this and the next section. Clearly

$$
F(s) \geq T_{0}^{n-1} \text { and thus } \quad k(t)=\frac{1}{F(s)} \leq T_{0}^{1-n},
$$

so $k(t)$ is bounded above. Also (23) holds with $\alpha_{1}=1$ and $\alpha_{2}=0$. Hence (a) holds.

As $F(s)$ is strictly increasing for $s \geq 0, F(s) s$ is also strictly increasing and so (b) holds.

To show (c), note that $n \geq 1$ and so

$$
\begin{aligned}
\frac{\mathrm{d}}{\mathrm{d} s}\left((F(s) s)^{1 / n}\right) & =\left(\left(s^{2}+T_{0}^{2}\right)^{(n-1) / 2} s\right)^{1 / n} \frac{n s^{2}+T_{0}^{2}}{n s\left(s^{2}+T_{0}^{2}\right)} \\
& \geq s \frac{n s^{2}+T_{0}^{2}}{n s\left(s^{2}+T_{0}^{2}\right)} \geq \frac{1}{n}
\end{aligned}
$$

Therefore, we may let $\gamma=n$ and thus $k(t) t$ is Hölder continuous with exponent $p-1$. 
To see that the monotonicity inequality (24) is satisfied, we define

$$
g(s)=C_{1}+C_{2}(F(s) s)^{2-p}-\frac{\mathrm{d}}{\mathrm{d} s}(F(s) s)
$$

and proceed to show that $g(s)$ is a non-negative function of $s$ for some constants $C_{1}$ and $C_{2}$.

First we note that $g(0) \geq 0$ for $C_{1}$ sufficiently large. This is so since $F(0)=0$ and

$$
\begin{aligned}
\frac{\mathrm{d}}{\mathrm{d} s}(F(s) s) & =\left(s^{2}+T_{0}^{2}\right)^{(n-3) / 2}(n-1) s^{2}+\left(s^{2}+T_{0}^{2}\right)^{(n-1) / 2} \\
& =\left(s^{2}+T_{0}^{2}\right)^{(n-3) / 2}\left(n s^{2}+T_{0}^{2}\right)
\end{aligned}
$$

and thus at $s=0$,

$$
g(0)=C_{1}-T_{0}^{n-1}
$$

which is non-negative for $C_{1} \geq T_{0}^{n-1}$. If the derivative of $g(s)$ is non-negative, then $g(s)$ is also non-negative.

Now

$$
\begin{aligned}
\frac{\mathrm{d} g}{\mathrm{~d} s}= & \frac{C_{2}(n-1)}{n}(F(s) s)^{1-p} \frac{\mathrm{d}}{\mathrm{d} s}(F(s) s)-\frac{\mathrm{d}^{2}}{\mathrm{~d} s^{2}}(F(s) s) \\
= & (n-1)\left(s^{2}+T_{0}^{2}\right)^{(n-5) / 2}\left(\frac{C_{2}(n-1)}{n} s^{2-1 / n}\left(s^{2}+T_{0}^{2}\right)^{(n+1) /(2 n)}\right. \\
& \left.+\frac{C_{2}}{n} s^{-1 / n}\left(s^{2}+T_{0}^{2}\right)^{1+(n+1) /(2 n)}-(n-3) s^{3}-3 s\left(s^{2}+T_{0}^{2}\right)\right) .
\end{aligned}
$$

To see that $\mathrm{d} g / \mathrm{d} s$ is non-negative, we need to show that the negative terms are dominated by the positive terms.

For the $-3 s\left(s^{2}+T_{0}^{2}\right)$ term, letting $C_{2} \geq 3 n$,

$$
\begin{aligned}
\frac{C_{2}}{n} s^{-1 / n}\left(s^{2}+T_{0}^{2}\right)^{1+(n+1) /(2 n)}-3 s\left(s^{2}+T_{0}^{2}\right) & \geq 3\left(s^{2}+T_{0}^{2}\right)\left(s^{-1 / n}\left(s^{2}+T_{0}^{2}\right)^{(n+1) /(2 n)}-s\right) \\
& \geq 3\left(s^{2}+T_{0}^{2}\right)\left(s^{-1 / n}(s)^{(n+1) / n}-s\right) \geq 0 .
\end{aligned}
$$

Next, if $n \leq 3$, the term $-(n-3) s^{3} \geq 0$, is positive and no bound on $C_{2}$ is needed. For $n \geq 3$, if we let $C_{2} \geq 3 n \geq n$, then

$$
\frac{C_{2}(n-1)}{n} s^{2-1 / n}\left(s^{2}+T_{0}^{2}\right)^{(n+1) /(2 n)}-(n-3) s^{3} \geq(n-3)\left(s^{2-1 / n} s^{2(n+1)(2 n)}-s^{3}\right) \geq 0 .
$$

Hence $F(s)$ possesses the properties (a)-(d) which implies that $k(t)$ possesses the properties (i)-(iv).

\section{Existence, UNIQUENESS AND ERROR ESTIMATES}

With the properties of $k(t)$ verified, we may apply the results in [3] to obtain the following lemmas and theorems.

Lemma 1. The operator $\mathcal{A}$ is continuous and monotone. More specifically, there exist constants $M_{1}, M_{2}>0$, $K \geq 0$ such that

and

$$
\|\mathcal{A} u-\mathcal{A} v\|^{*} \leq M_{2}\|u-v\|^{p-1}
$$

for all $u, v$ in $V$.

$$
M_{1}\|u-v\|^{2} \leq(\mathcal{A} u-\mathcal{A} v, u-v)\left(1+K(\|u\|+\|v\|)^{2-p}\right)
$$


Lemma 2. The weak solution $u$ and the finite element approximation $u_{h}$ are uniformly bounded above, i.e. there exists a constant $C$, independent of $u$ and $u_{h}$, such that

$$
\|u\|,\left\|u_{h}\right\| \leq C .
$$

Theorem 1. The weak problem (21) is well posed.

Theorem 2. There exists a constant $C>0$, independent of $u$ and $u_{h}$, such that

$$
\left\|u-u_{h}\right\| \leq C \inf \left\{\left\|u-v_{h}\right\|^{p / 2} ; v_{h} \in V_{h}\right\}
$$

Corollary 1. If the weak solution $u \in W^{2, p}(\Omega) \cap V$ and $V_{h} \subset V$ is the space of linear finite elements, then

$$
\left\|u-u_{h}\right\|=O\left(h^{p / 2}\right) .
$$

The interested reader may refer to [3] for proofs of the above results.

Consequently, from Theorem 2 and interpolation theory, we see that if the weak solution $u$ is minimally regular, we have:

Theorem 3. If the weak solution $u \in W^{r, p} \cap V$ for some $r>1$ then the finite element approximation $u_{h}$ converges to $u$ strongly in $V$ as the mesh parameter $h$ tends to zero.

\section{Optimal estimates}

As may be seen in Corollary 1, the error estimates derived in the previous section are suboptimal when compared with the well known estimates for analogous linear problems. It is of interest to know if one can establish optimal error estimates for the finite element approximations by imposing additional conditions. In this section we explore this issue by examining the quasi-norm approach of Liu and Barrett [12] and the method of Johnson and Thomee [11] generalized by Chow [4].

\subsection{Quasi-norm}

In [12], Liu and Barrett provided a framework for deriving optimal order error estimates based on the socalled quasi-norm. Let $u$ be the weak solution of (21). For any $v \in V$ and any $\delta \geq-2$, the quasi-norm of $v \in V$ is defined as

$$
\|v\|_{(p, \delta)}^{2}=\int_{\Omega}(|\nabla u|+|\nabla v|)^{p-2-\delta}|\nabla v|^{2+\delta} \mathrm{d} x \mathrm{~d} z .
$$

For the results of [12] to be applicable to the glacier model, we need to show that the following assumptions hold for the gradient dependent coefficient $k$.

There exist positive constants $C, M, K_{1}, K_{2}$ and $p^{+} \in[p, 2]$ such that

$$
\begin{aligned}
& \left|\frac{\mathrm{d}}{\mathrm{d} t}(k(t) t)\right| \leq C t^{p^{+}-2} \quad \text { for all } t>0 \\
& \left(K_{1}+K_{2} t^{2-p}\right) \frac{\mathrm{d}}{\mathrm{d} t}(k(t) t) \geq M \quad \text { for all } t>0 .
\end{aligned}
$$

Since (A2) is identical to the monotonicity condition (iv) it follows that (A2) holds for Glen's flow law $F(s)$.

The condition (A1) for $p^{+}=p$ may be restated in terms of $F(s)$ as

$$
C(F(s) s)^{2-p} \leq \frac{\mathrm{d}}{\mathrm{d} s}(F(s) s) \text { for all } s>0 .
$$

As $n=1 /(p-1)$, so

$$
(F(s) s)^{2-p}=\left(\left(s^{2}+T_{0}^{2}\right)^{\frac{n-1}{2}} s\right)^{\frac{n-1}{n}} \leq\left[\left(s^{2}+T_{0}^{2}\right)^{\frac{n-1}{2}}\left(s^{2}+T_{0}^{2}\right)^{\frac{1}{2}}\right]^{\frac{n-1}{n}}=\left(s^{2}+T_{0}^{2}\right)^{\frac{n-1}{2}} .
$$


From (25),

$$
\frac{\mathrm{d}}{\mathrm{d} s}(F(s) s)=\left(s^{2}+T_{0}^{2}\right)^{\frac{n-3}{2}}\left(n s^{2}+T_{0}^{2}\right) \geq\left(s^{2}+T_{0}^{2}\right)^{\frac{n-3}{2}}\left(s^{2}+T_{0}^{2}\right)=\left(s^{2}+T_{0}^{2}\right)^{\frac{n-1}{2}}
$$

and thus condition (A1) is satisfied for $p=1+\frac{1}{n}$.

In fact, we have

$$
\frac{\mathrm{d}}{\mathrm{d} s}(F(s) s) \geq T_{0}^{n-1}>0 .
$$

So condition (A1) is satisfied with $p^{+}=2$.

Consequently, we have the following results for the finite element approximation $u_{h}$ :

Theorem 4 (see Th. $3.1 \mathrm{in}[12]$ ). If the weak solution $u$ of (21) belongs to $W^{1, p^{+}}(\Omega) \cap V$, then for any $\delta \in(-2,0]$ and $v_{h} \in V_{h}$,

$$
\left\|u-u_{h}\right\| \leq C\left\|u-v_{h}\right\|_{\left(p^{+}, \delta\right)}
$$

Corollary 2 (see Cor. 3.1 in [12]). For piecewise linear finite element space $V_{h} \subset V$, if $u \in W^{2, p^{+}} \cap V$,

$$
\left\|u-u_{h}\right\| \leq C h^{p^{+} / 2}
$$

so, in particular, for the glacier model (15)-(17), with $u \in H^{2}(\Omega) \cap V$,

$$
\left\|u-u_{h}\right\| \leq C h .
$$

\subsection{An alternative approach}

Instead of deriving the optimal bound using the quasi-norm, we can also apply the result of Chow [4]. This approach requires a $W^{1, \infty}(\Omega)$ regularity assumption on the weak solution as well as restriction to using linear elements but has the added advantage of allowing us to prove uniform pointwise boundedness of the gradient of the finite element approximations. This, in turn, allows us to prove the convergence of the successive iteration solution algorithm as seen in Section 5 following.

As indicated in [4], the results then rely on the requirement that the function $F(s)$ possesses the following properties.

(I) $F$ is bounded below by some positive constant;

(II) for each nonnegative $s_{1}, s_{2}$ with $s_{1}<M$, there exists a positive constant $\gamma<1$ such that

$$
s_{1}\left|F\left(s_{1}\right)-F\left(s_{2}\right)\right| \leq \gamma\left|F\left(s_{1}\right) s_{1}-F\left(s_{2}\right) s_{2}\right| ;
$$

(III)

$$
\int_{\Omega} k^{-\frac{p}{2-p}}\left(\left|\nabla u_{1}\right|\right) \mathrm{d} x \leq C
$$

for some constant $C$ independent of $u_{h}$.

Clearly $F(s)=\left(s^{2}+T_{0}^{2}\right)^{\frac{n-1}{2}} \geq T_{0}^{n-1}>0$ is bounded below so (I) holds. For (II), (26) holds when $s_{1}=0$ or $s_{1}=s_{2}$ or $n=1$. For $s_{2} \equiv 0, s_{1} \leq M$

$$
\frac{s\left|F\left(s_{1}\right)-F\left(s_{2}\right)\right|}{\left|F\left(s_{1}\right) s_{1}-F\left(s_{2}\right) s_{2}\right|}=\frac{s_{1}\left(\left(s_{1}^{2}+T_{0}^{2}\right)^{\frac{n-1}{2}}-\left(T_{0}^{2}\right)^{\frac{n-1}{2}}\right)}{s_{1}\left(s_{1}^{2}+T_{0}^{2}\right)^{\frac{n-1}{2}}} \leq 1-\left(\frac{T_{0}^{2}}{M^{2}+T_{0}^{2}}\right)^{\frac{n-1}{2}} \leq \gamma<1
$$

for some constant $\gamma$. 
Let

$$
S=\sup _{\substack{s_{1} \neq s_{2} \\ 0<s_{1} \leq M}} \frac{s_{1}\left|F\left(s_{1}\right)-F\left(s_{2}\right)\right|}{\left|F\left(s_{1}\right) s_{1}-F\left(s_{2}\right) s_{2}\right|} .
$$

Then (26) holds if we can show that $S \leq \gamma$ for some $\gamma<1$. Now, let

$$
S=\max \left(\sup _{0<s_{2}<s_{1} \leq M} S_{1}, \sup _{\substack{s_{1}<s_{2}, s_{1} \leq M}} S_{2}\right)
$$

where

$$
S_{1}=\frac{s_{1}\left(F\left(s_{1}\right)-F\left(s_{2}\right)\right)}{F\left(s_{1}\right) s_{1}-F\left(s_{2}\right) s_{2}}, \quad S_{2}=\frac{s_{1}\left(F\left(s_{2}\right)-F\left(s_{1}\right)\right)}{F\left(s_{2}\right) s_{2}-F\left(s_{1}\right)\left(s_{1}\right)} .
$$

Let $s_{1}=s$ be fixed and let $0<\kappa<1$ be such that $s_{2}=\kappa s$. Then

$$
S_{1}=\frac{\left(\left(s^{2}-T_{0}^{2}\right)^{\frac{n-1}{2}}-\left(\kappa^{2} s^{2}+T_{0}^{2}\right)^{\frac{n-1}{2}}\right)}{\left(s^{2}+T_{0}^{2}\right)^{\frac{n-1}{2}}-\kappa\left(\kappa^{2} s^{2}+T_{0}^{2}\right)^{\frac{n-1}{2}}}
$$

Suppose $n>1$ is odd. We write $m=(n-1) / 2$ and expand each term of $S_{1}$ using the binomial theorem to get

$$
\begin{aligned}
S_{1}= & \frac{\sum_{i=0}^{m}\left(\begin{array}{c}
m \\
i
\end{array}\right) s^{2(m-i)} T_{0}^{2 i}-\sum_{i=0}^{m}\left(\begin{array}{c}
m \\
i
\end{array}\right) \kappa^{2(m-i)} \kappa s^{2(m-i)} T_{0}^{2 i}}{\sum_{i=0}^{m}\left(\begin{array}{c}
m \\
i
\end{array}\right) s^{2(m-i)} T_{0}^{2 i}-\sum_{i=0}^{m}\left(\begin{array}{c}
m \\
i
\end{array}\right) \kappa^{2(m-i)+1} s^{2(-i)} T_{0}^{2 i}} \\
\leq & \frac{\sum_{i=0}^{m-1}\left(\begin{array}{c}
m \\
i
\end{array}\right) s^{2(m-i)} T_{0}^{2 i} \sum_{j=0}^{2(m-i)} \kappa^{j}}{T_{0}^{2}+\sum_{i=0}^{m-1}\left(\begin{array}{c}
m \\
i
\end{array}\right) s^{2(m-i)} T_{0}^{2 i} \sum_{j=0}^{2(m-i)} \kappa^{j}} \\
\equiv & \frac{h(\kappa, s)}{T_{0}^{2}+h(\kappa, s)} .
\end{aligned}
$$

Recall that if $h(x)$ is a monotone increasing differentiable function of $x$ and $a>0$ is a constant, then $h(x) /(a+$ $h(x))$ is also a monotone increasing function of $x$. As $h(\kappa, s)$ is an increasing function in $\kappa$ and in $s$, with $0<\kappa<1$ and $s \leq M$, hence

$$
S_{1} \leq \frac{h(\kappa, s)}{T_{0}^{2}+h(\kappa, s)} \leq \frac{h(1, s)}{T_{0}^{2}+h(1, s)} \leq \frac{h(1, M)}{T_{0}^{2}+h(1, M)} \leq \gamma<1 .
$$

If $n$ is even, let $m=n-1$ and we have

$$
\begin{aligned}
S_{1} & =\frac{\left(s^{2}+T_{0}^{2}\right)^{m}-\left(\kappa^{2} s^{2}+T_{0}^{2}\right)^{m}}{\left(s^{2}+T_{0}^{2}\right)^{m}-\kappa^{2}\left(\kappa^{2} s^{2}+T_{0}^{2}\right)^{m}} \cdot \frac{\left(s^{2}+T_{0}^{2}\right)^{\frac{n-1}{2}}+\kappa\left(s^{2}+T_{0}^{2}\right)^{\frac{n-1}{2}}}{\left(s^{2}+T_{0}^{2}\right)^{\frac{n-1}{2}}+\left(s^{2}+T_{0}^{2}\right)^{\frac{n-1}{2}}} \\
& \leq \frac{\left(s^{2}+T_{0}^{2}\right)^{m}-\left(\kappa^{2} s^{2}+T_{0}^{2}\right)^{m}}{\left(s^{2}+T_{0}^{2}\right)^{m}-\kappa^{2}\left(\kappa^{2} s^{2}+T_{0}^{2}\right)^{m}}
\end{aligned}
$$


since the quotient involving the $\frac{n-1}{2}$ power is bounded above by 1 . Expanding using the binomial theorem we may proceed as before to get $S_{1} \leq \gamma<1$. To treat the $S_{2}$ term, we again fix $s_{1}=s$ and let $s_{2}=\kappa s$ with $\kappa>1$. Now

$$
\begin{aligned}
\frac{1}{S_{2}} & =\frac{\kappa\left(\kappa^{2} s^{2}+T_{0}^{2}\right)^{\frac{n-1}{2}}-\left(s^{2}+T_{0}^{2}\right)^{\frac{n-1}{2}}}{\left(\kappa^{2} s^{2}+T_{0}^{2}\right)^{\frac{n-1}{2}}-\left(s^{2}+T_{0}^{2}\right)^{\left(\frac{n-1}{2}\right)}} \\
& =1+\frac{(\kappa-1)}{1-\left(\frac{s^{2}-T_{0}^{2}}{\kappa^{2} s^{2}+T_{0}^{2}}\right)^{\left(\frac{n-1}{2}\right)}} .
\end{aligned}
$$

Recall that $\left(1-y^{\alpha}\right) \leq(1+\alpha)(1-y)$ for all $y \in(0,1]$ and $\alpha>0$, so

$$
\begin{aligned}
\frac{1}{S_{2}} & \geq 1+\frac{\kappa-1}{\left(1+\frac{n-1}{2}\right)\left(1-\frac{s^{2}+T_{0}^{2}}{\kappa^{2} s^{2}+T_{0}^{2}}\right)}=1+\frac{2}{n+1} \frac{\kappa^{2} s^{2}+T_{0}^{2}}{(\kappa+1) s^{2}} \\
& \geq 1+\frac{2}{n+1} \frac{s^{2}+T_{0}^{2}}{(\kappa+1) s^{2}} \geq 1+\frac{1}{n+1}\left(1+\left(\frac{T_{0}}{M}\right)^{2}\right)
\end{aligned}
$$

and thus $S_{2} \leq \gamma<1$.

Consequently

$$
S=\max \left(S_{1}, S_{2}\right) \leq \gamma<1
$$

for some constant $\gamma<1$ and so condition (II) holds and we have the following result:

Lemma 3. Let $I^{2}=\int_{\Omega} k\left(\left|\nabla u_{h}\right|\right)\left|\nabla\left(u-u_{h}\right)\right|^{2} \mathrm{~d} x$ with $u$ and $u_{h}$ being the weak solution of (21) and the finite element approximation (22) respectively. Then there exists a constant $C>0$ such that for all $w_{h} \in V_{h} \cap H^{1}(\Omega)$,

$$
I \leq C\left\|\nabla\left(u-w_{h}\right)\right\|_{L^{2}(\Omega)} .
$$

The proof of this lemma is similar to that found in [4].

Finally, to verify condition (III), (27), first note that $\frac{p}{2-p}=(n+1) /(n-1)$ and so

$$
\begin{aligned}
F(s)^{\frac{p}{2-p}} & =\left(s^{2}+T_{0}^{2}\right)^{\frac{n+1}{2}} \leq 2^{\frac{n-1}{2}}\left(s^{n+1}+T_{0}^{n+1}\right) \\
& \leq 2^{\frac{n-1}{2}} T_{0}^{n-1}+2^{\frac{n-1}{2}}\left[\left(s^{2}+T_{0}^{2}\right)^{\frac{n-1}{2}} s\right]^{p} \leq 2^{\frac{n-1}{2}} T_{0}^{n-1}+2^{\frac{n-1}{2}}(F(s) s)^{p} .
\end{aligned}
$$

By making use of the relation $F(s) k(t)=1$ where $s=k(t) t$ and $t=F(s) s$, we have

$$
k(t)^{-p /(2-p)} \leq 2^{\frac{n-1}{2}} T_{0}^{n-1}+2^{\frac{n-1}{2}} t^{p} .
$$

Setting $t=\left|\nabla u_{h}\right|$ and applying Lemma 2, we see that condition (III), (27), is satisfied:

$$
\int_{\Omega} k\left(\left|\nabla u_{h}\right|\right)^{-p /(2-p)} \mathrm{d} x \leq C_{1}+C_{2}\left\|u_{h}\right\| \leq C .
$$

Hence we have the following results, the proofs of which may be found in [4].

Theorem 5. Suppose the weak solution u of (21) belongs to the space $W^{1, \infty}(\Omega) \cap V$, then for some $C>0$, and for all $w_{h} \in V_{h} \cap H^{1}(\Omega)$,

$$
\left\|u-u_{h}\right\| \leq C\left\|\nabla\left(u-w_{h}\right)\right\|_{L^{2}(\Omega)} .
$$


Corollary 3. If $u \in H^{2}(\Omega) \cap W^{1, \infty}(\Omega) \cap V$, and $v_{h}$ is the subspace of linear finite elements of $V$, then

$$
\left\|u-u_{h}\right\|=\mathcal{O}(h) .
$$

Thus we have the optimal error estimate. Furthermore, $\nabla u_{h}$ is uniformly pointwise bounded.

Lemma 4. If the weak solution $u$ of (21) is in the space $H^{2}(\Omega) \cap W^{1, \infty}(\Omega) \cap V$, then there is a constant $C$ independent of $u_{h}$ such that for any $0<h<1$,

$$
\left\|\nabla u_{h}\right\|_{L \infty(\Omega)} \leq C .
$$

Theorem 6. Suppose the weak solution $u$ of (21) belongs to the space $H^{2}(\Omega) \cap W^{1, \infty}(\Omega) \cap V$, and $V_{h}$ is the linear finite element space in $V$, then

$$
\left\|u-u_{h}\right\|_{1,2}=\mathcal{O}(h)
$$

\section{SucCessive approximation}

Another unsolved issue in [7] is the theoretical analysis of the convergence of the successive iteration:

(1) Select an initial guess $u_{h}^{(0)}$;

(2) for $i=0,1,2, \ldots$ until convergence, solve the linear problem

$$
\int_{\Omega} k\left(\left|\nabla u_{h}^{(i)}\right|\right) \nabla u_{h}^{(i+1)} \cdot \nabla w_{h} \mathrm{~d} x \mathrm{~d} z=\int_{\Omega} f w_{h} \mathrm{~d} x \mathrm{~d} z
$$

for all $w_{h} \in V_{h}$.

We now prove the convergence of the above algorithm for linear finite element approximations.

We assume that the weak solution $u$ of $(21)$ is in the space $H^{2}(\Omega) \cap W^{1, \infty}(\Omega) \cap V$. By Lemma 4 , the linear finite element approximations are pointwise uniformly bounded for $0<h<1$. Let $\|\nabla u\|_{L^{\infty}(\Omega)},\left\|\nabla u_{h}\right\|_{L^{\infty}(\Omega)} \leq M_{\infty}$,

$$
K=\left\{v \in V \cap H^{1}(\Omega) ; \quad\|\nabla v\|_{L^{\infty}} \leq M_{\infty}\right\}
$$

and let

$$
K_{h}=\left\{v_{h} \in V_{h} \cap H^{1}(\Omega) ; \quad\left\|\nabla v_{h}\right\|_{L^{\infty}} \leq M_{\infty}\right\} .
$$

Then the constrained minimization problems

$$
\min \{J(v) ; \quad v \in K\}
$$

and

have solutions $u$ and $u_{h}$ respectively.

$$
\min \left\{J\left(v_{h}\right) ; \quad v_{h} \in K_{h}\right\}
$$

Let $u_{0}$ be some initial guess of the weak solution $u$. For each $i \geq 0$, the solution of the successive approximation scheme is obtained by solving the minimization problem

$$
\min \left\{\frac{1}{2} B\left(u_{h}^{(i)} ; v_{h}, v_{h}\right)-\left(f, v_{h}\right) ; \quad v_{h} \in K_{h}\right\} .
$$

where $B(u ; v, w)=\int_{\Omega} k(|\nabla u|) \nabla v \cdot \nabla w \mathrm{~d} x$.

Now for $0 \leq t \leq M_{\infty}, 0 \leq s \leq k\left(M_{\infty}\right) M_{\infty}=M_{0}$

$$
A T_{0}^{n-1} \leq F(s) \leq A\left(M_{0}^{2}+T_{0}^{2}\right)^{\frac{n-1}{2}}
$$


and so $T_{0}^{1-n} / A \geq k(t) \geq\left(M_{0}^{2}+T_{0}^{2}\right)^{-(n-1) / 2} / A$. Thus $k(t)$ is uniformly bounded above and below and is decreasing. Furthermore,

$$
\begin{aligned}
\frac{\mathrm{d}}{\mathrm{d} s} F(s) s & =F(s)+F^{\prime}(s) s \\
& \leq A\left(M_{0}^{2}+T_{0}^{2}\right)^{\frac{n-1}{2}}+\frac{n-1}{2} A\left(M_{0}^{2}+T_{0}^{2}\right)^{\frac{n-1}{3}}\left(2 M_{0}\right) \cdot M_{0}
\end{aligned}
$$

so $\frac{\mathrm{d}}{\mathrm{d} t} k(t) t \geq a>0$ for some constant $a$, i.e. $\frac{\mathrm{d}}{\mathrm{d} t} k(t) t$ is bounded below by some positive constant. Consequently, using ideas developed in [10], we have

Theorem 7. For fixed $h$, the solutions $u_{h}^{(i)}$ of the successive iteration algorithm converge to the finite element solution $u_{h}$ as $i \rightarrow \infty$.

Proof. The bilinear form $(v, w) \mapsto B(u ; v, w)$ is symmetric and since $k(t)$ is bounded above and below for bounded $t$, then there exists constants $\delta_{0}, \delta_{1}>0$ such that

$$
|B(u ; v, w)| \leq \delta_{1}\|v\|_{H^{1}}\|w\|_{H^{1}} \text { for all } u \in K_{h}, v, w \in V_{h} \cap H^{1}
$$

and

$$
B(u ; v-w, u-w) \geq \delta_{0}\|v-w\|_{H^{1}} \text { for all } u, v, w \in K_{h} .
$$

Furthermore, $F(s)$ is an increasing function and hence $k(t)$ is a decreasing function, so

$$
\int_{t_{1}}^{t_{2}} k(t) t \mathrm{~d} t \geq \frac{1}{2} k\left(t_{1}\right)\left(t_{2}^{2}-t_{1}^{2}\right) \text { for all } t_{1}, t_{2} \geq 0
$$

and thus for all $u, v \in K_{h}$

$$
\int_{\Omega} \int_{0}^{|\nabla v|} k(t) t \mathrm{~d} t-\int_{0}^{|\nabla u|} k(t) t \mathrm{~d} t \mathrm{~d} x \mathrm{~d} z \geq \frac{1}{2} B(u ; v, v)-B(u ; u, u) .
$$

It follows from a standard convergence theorem, Theorem 25.L in [14], that we have the convergence of the successive approximation iterations $u_{h}^{(i)}$ to $u_{h}$ as $i \rightarrow \infty$.

We remark that the above theorem will hold for finite elements of any degree if one can show that the finite element approximations to (21) are uniformly bounded in the $W^{1, \infty}$ norm.

\section{Numerical Results}

In this section we briefly describe the implementation of a finite element scheme for solving the glacier model based on Glen's flow law and conduct numerical experiments designed to study the convergence rates as the mesh is refined. These rates are then compared with the theoretical rates derived in the previous sections. To demonstrate the rates of convergence we consider both the example in [7] in which a segment of the base of the glacier is free to slip, and an example with solution of higher regularity. In both cases there are no known analytic solutions. However, for the first case, the adjacency of the stick-slip zones implies singularities at the respective stick-slip points on the base. We also consider the use of adaptive mesh refinement to obtain accurate and reliable solutions, especially in the presence of such singularities.

In all cases shown here, the Glen's flow law parameters are $A=1, n=2.0$ and $T_{0}^{2}=0.1$. For this set of parameters, the form of the function $k$ is not known explicitly and is computed using the Glen's flow law function $F$. 


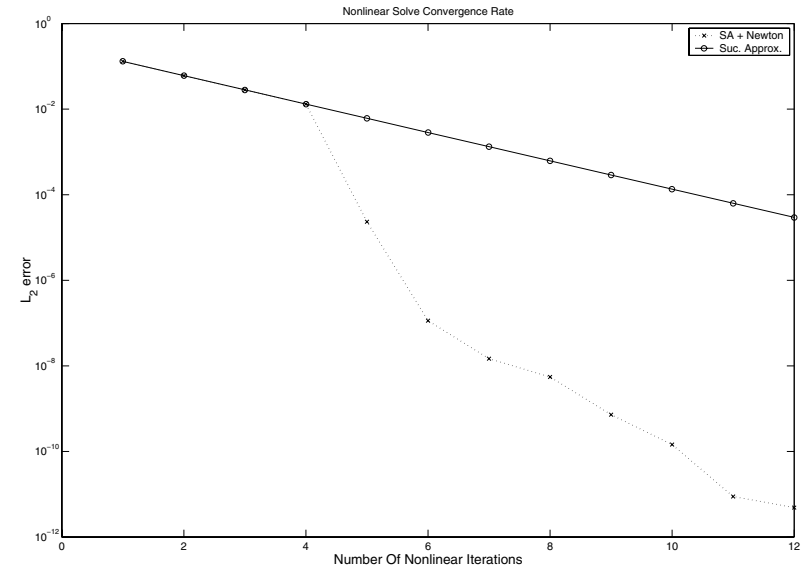

FIGURE 1. Convergence rates for pure successive approximation and combined successive approximation - Newton's method.

\subsection{Solution method}

The finite element model employs continuous bilinear or biquadratic approximation on quadrilateral elements and the adaptive mesh refinement strategy (AMR) proceeds by using an error indicator based on element edgeinterface jumps to locally subdivide selected elements into quartets of subelements. The conformity of the approximation is enforced across the interface between refined and unrefined elements using the standard 2:1 refinement rule [2].

A combination of successive approximation and Newton's method is used to solve the nonlinear part of the problem. Since successive approximations is often more robust than Newton iteration, and we have demonstrated convergence of successive approximations, several successive approximation iterations are first applied and Newton's method is then used for faster solution convergence. The convergence rates are shown in Figure 1.

The solutions were calculated on a sequence of nested refined grids, both adaptive and uniform. Letting $u_{n}$ denote to the solution of the grid after $n$ refinements, then the error is approximated using $e_{n} \approx u_{n}-u_{n-1}$.

\subsection{Case 1 - Singular solution}

We apply both uniform and adaptive refinement strategies to the singular problem studied in [7] in which a segment of the base is allowed to slip. The problem is solved on the domain $[0,60] \times[0,2]$ with a no slip condition, $u=0$, on most of the base $(0<x<28$ and $32<x<60)$, and with Neumann boundary condition, $\nabla u \cdot \nu=0$, on the top and small section of the base $(28<x<32)$. The initial mesh spacings were $\Delta x \approx 3.1$, $\Delta y=0.2$. The inflow/outflow velocity profiles are those used in [7]: $f(y)=\frac{1}{32} y^{4}+\frac{1}{4} y^{3}-\frac{T_{0}^{2}+3}{4} y^{2}+\left(T_{0}^{2}+1\right) y$.

A contour plot of the solution $u$ is shown in Figure 2. Note the strong singularities at the stick slip points. The convergence plots for this case are illustrated in Figure 3. The rates of convergence are given by the slopes of the graphs and are summarized in Table 1.

The effect of the singularity on the rate of convergence is illustrated by the uniform refinement studies which show a reduced rate of convergence, $h^{0.3}$ in the $W^{1,2}$ norm and $h^{1}$ in the $L^{2}$ norm. These rates of convergence appear to be independent of the degree of the finite element basis. The AMR scheme refines the grid adaptively towards the singularity and recovers an improved rate of convergence, as shown in Figure 3 and Table 1. 


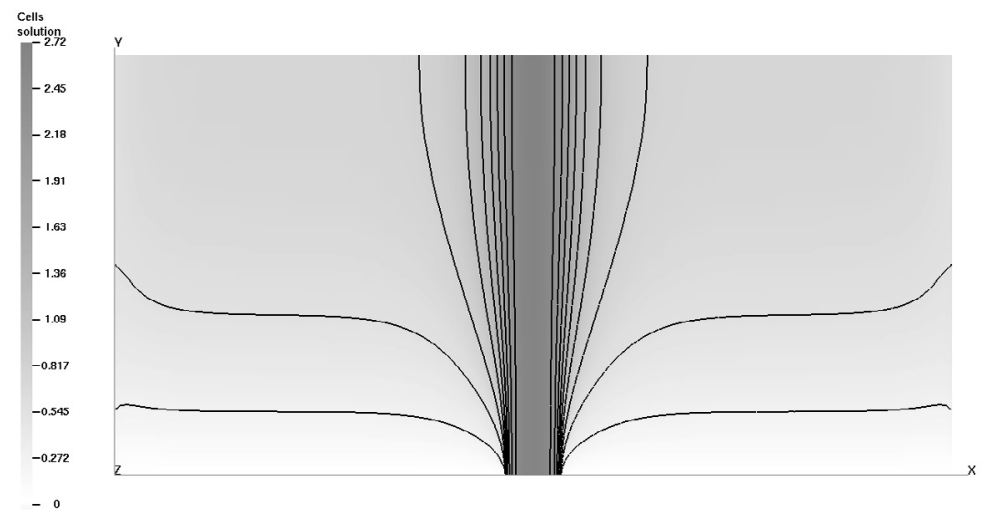

Figure 2. Contours of $u$ for the singular solution.
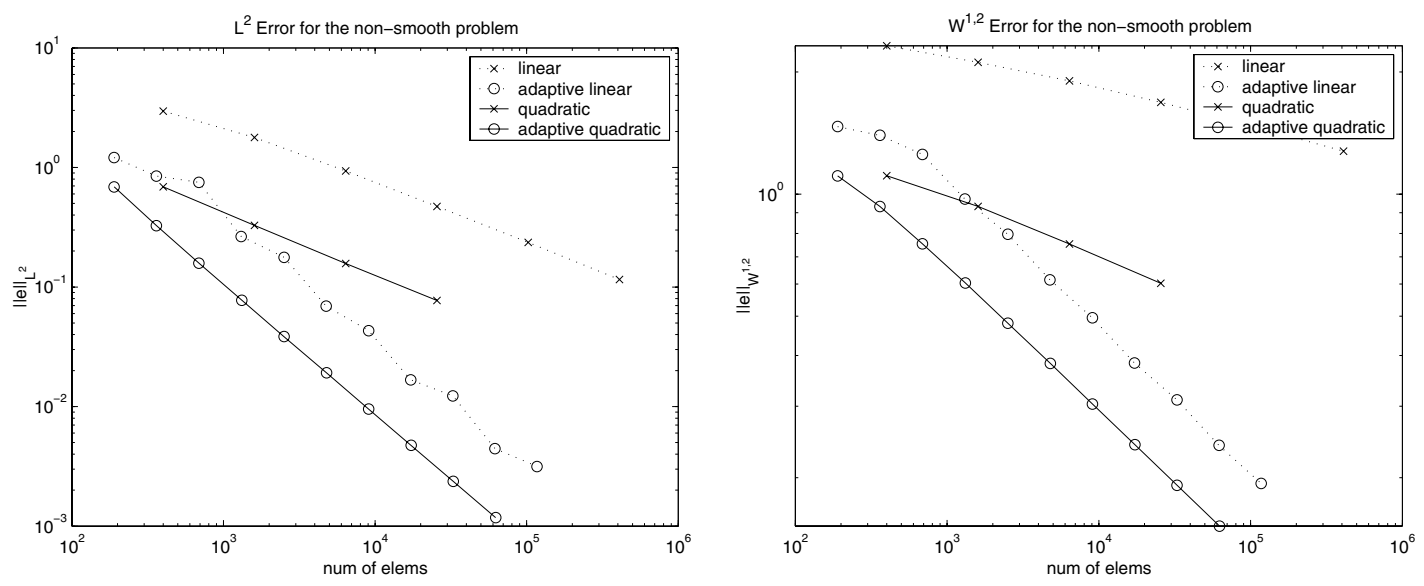

FiguRE 3. $L^{2}$ and $W^{1,2}$ error for the singular problem.

TABLE 1. Summary of convergence rates for the non-smooth and smooth problems.

\begin{tabular}{|c|c|c|c|c|}
\hline & \multicolumn{2}{|c|}{ Singular } & \multicolumn{2}{c|}{ Smooth } \\
& Linear (adapt.) & Quadratic (adapt.) & Linear & Quadratic \\
\hline$W^{1,2}$ & $h^{0.2}\left(h^{0.7}\right)$ & $h^{0.3}\left(h^{0.7}\right)$ & $h^{1}$ & $h^{2}$ \\
$L^{2}$ & $h^{1}\left(h^{2}\right)$ & $h^{1}\left(h^{2}\right)$ & $h^{2}$ & $h^{3}$ \\
\hline
\end{tabular}

\subsection{Case 2 - Smooth solution}

In the previous example, the presence of singularities is due to the incompatibility of the stick-slip boundary conditions. We can avoid such singularities by using compatible Dirichlet boundary conditions instead. Numerical experiments seem to indicate that the solution of the resulting problem is sufficiently smooth.

The particular problem solved was in the domain $[0,10] \times[0,2]$ with no slip condition on the base $(u=0$ on $y=0)$, prescribed inflow and outflow velocity profiles $(u=f(y)$ on $x=0$ and $x=L)$ and specified velocity on the surface $(u=g(x)$ on $y=2)$. While this is not a physically based example, it is similar to the example considered in [7] or 6.2 . 

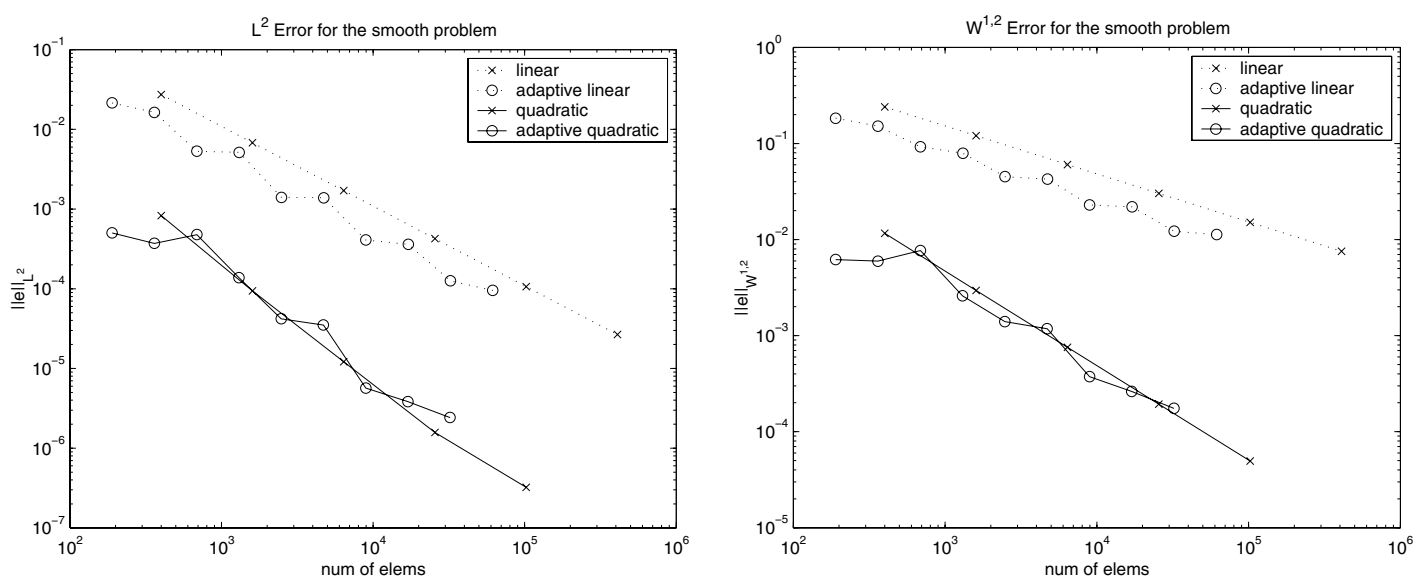

FiguRE 4. $L^{2}$ and $W^{1,2}$ error for the smooth problem.

The inflow and outflow velocity profiles were the same as in case 1 . The velocity at the surface is chosen to be $g(x)=0.5+T_{0}^{2}+\frac{x(10-x)}{20}$ which matches the inflow/outflow velocities at the edges.

In the numerical studies, the problem was first solved on a coarse mesh with characteristic mesh sizes $\Delta x=0.5, \Delta y=0.2$ and subsequently solved on uniformly refined meshes. The convergence behavior is illustrated by the log-log plot of the $L^{2}$ and $W^{1,2}$ norms of the error versus number of elements as seen in Figure 4.

The rate of convergence is given by the slope of the graph and confirms the optimal rate predicted by the theory. As one may see from Table 1, the rate of convergence matches the theoretical rate.

The figure also shows convergence results for the same problem using AMR. In the absence of a singularity, the adaptive strategy leads to a fairly uniformly refined mesh and the rates of convergence obtained are similar to those for uniform meshes.

\section{CONCLUSION}

We have shown that the finite element solution error for the simplified glaciology problem (9), (13), (14) is bounded by the projection of the weak solution to the finite element space under the quasi-norm. With reasonable regularity assumptions on the weak solution, the error estimates are optimal for various degree of finite elements. For linear elements, we have used an alternate technique to obtain an optimal energy error estimate and have shown that the finite element approximations are uniformly bounded in the $W^{1, \infty}$-norm. The last result allowed us to prove the convergence of a modified successive approximations procedure.

In our numerical experiments, we have employed both bilinear and biquadratic elements to confirm the optimality of the error estimates when the exact solution is sufficiently smooth. We also showed the effectiveness of adaptivity for this glaciology problem with a stick-slip singularity. We also demonstrated that the solution procedure may be accelerated by coupling Newton's method with successive approximations. It seems likely that convergence proofs of the successive approximations procedure and the Newton's method for higher order finite elements may be obtained with the aid of the quasi-norms.

Remark. A related paper by Glowinski and Rappaz [9] has recently appeared in this journal and come to our attention. Both papers made use of the quasi-norm technique in deriving optimal error estimates for the finite element solutions. In this paper we also proved the convergence of the successive approximations and studied the effectiveness of adaptivity in treating problems with a stick-slip singularity. 


\section{REFERENCES}

[1] H. Blatter, Velocity and stress fields in grounded glaciers: A simple algorithm for including deviatoric stress gradients. J. Glaciology 41 (1995) 333-344.

[2] G.F. Carey, Computational Grids: Generation, Adaptation and Solution Strategies. Taylor \& Francis (1997).

[3] S.-S. Chow, Finite element error estimates for nonlinear elliptic equations of monotone type. Numer. Math. 54 (1989) 373-393.

[4] S.-S. Chow, Finite element error estimates for a blast furnace gas flow problem. SIAM J. Numer. Analysis 29 (1992) 769-780.

[5] S.-S. Chow and G.F. Carey, Numerical approximation of generalized Newtonian fluids using Heindl elements: I. Theoretical estimates. Internat. J. Numer. Methods Fluids 41 (2003) 1085-1118.

[6] J. Colinge and H. Blatter, Stress and velocity fields in glaciers: Part I. Finite-difference schemes for higher-order glacier models. J. Glaciology 44 (1998) 448-456.

[7] J. Colinge and J. Rappaz, A strongly nonlinear problem arising in glaciology. ESAIM: M2AN 33 (1999) 395-406.

[8] J.W. Glen, The Flow Law of Ice, Internat. Assoc. Sci. Hydrology Pub. 47, Symposium at Chamonix 1958 - Physics of the Movement of the Ice (1958) 171-183.

[9] R. Glowinski and J. Rappaz, Approximation of a nonlinear elliptic problem arising in a non-Newtonian fluid flow model in glaciology. ESAIM: M2AN 37 (2003) 175-186.

[10] W. Han, J. S $\phi$ ren and I. Shimansky, The Kačanov method for some nonlinear problems. Appl. Num. Anal. 24 (1997) 57-79.

[11] C. Johnson and V. Thomee, Error estimates for a finite element approximation of a minimal surface. Math. Comp. 29 (1975) 343-349.

[12] W.B. Liu and J.W. Barrett, Finite element approximation of some degenerate monotone quasilinear elliptic systems. SIAM J. Numer. Analysis 33 (1996) 98-106.

[13] W.S.B. Patterson, The Physics of Glaciers, 2nd edition. Pergamon Press (1981).

[14] E. Zeidler, Nonlinear Functional Analysis and Its Applications II/B. Nonlinear Monotone Operators, Springer-Verlag (1990).

To access this journal online: www.edpsciences.org 